\title{
Avances en la experiencia peruana de la implementación de la gestión por resultados
}

\section{Advances in the Peruvian experience of the implementation of management by results}

\section{Carlos Ricse}

En el Perú, las reformas se producen siempre con retraso de alrededor de una década en relación con países de equivalente ingreso medio. Ello le brinda la oportunidad de aprender de los procesos ocurridos en aquellos países y evitar cometer los errores de la implementación progresiva, aunque no siempre con éxito. Una buena parte de esta importación de la agenda pública ha sido impulsada por los organismos multilaterales, en el marco de operaciones de endeudamiento vinculado a matrices de reforma sectorial. Fue así con las reformas fiscales y tributarias en los noventa; los programas sociales, la inversión pública y la descentralización en la primera mitad de la década de 2000; y, más recientemente, con la mejora de la calidad del gasto público y la protección social.

La calidad del gasto público puede entenderse como la capacidad que tiene el Estado, a través del presupuesto público, de proveer bienes y servicios con estándares de calidad, por medio de los cuales se logren resolver los problemas de la población, en particular de los pobres y excluidos. Definida así la calidad del gasto público, destaca el principio de eficacia, definida como la capacidad de conseguir los propósitos buscados.

El otro principio que resalta en la definición de la calidad del gasto público es la eficiencia, definida como la capacidad de usar óptimamente los recursos escasos con que se cuenta, de tal modo que pueda maximizarse el impacto de la acción pública. Cabe señalar que la eficiencia en el gasto público no debe ser entendida como la capacidad de ahorrar recursos financieros, pues existe el riesgo que con el ahorro se esté afectando la posibilidad de conseguir los resultados buscados. En síntesis, la calidad del gasto público implica atender simultáneamente a los principios de eficacia y eficiencia para conseguir los mayores resultados o impacto en la población.

En la experiencia de los últimos 7 años de implementación del Presupuesto por Resultados en el Perú, se debe reconocer que aún cuando eficacia y eficiencia deben ser consideradas simultáneamente en el gasto público, la eficacia debe tener mayor prioridad. El Estado debe honrar su compromiso de servicio con la Sociedad a quien se debe, no

*Dirección de correspondencia [Correspondence address]: Carlos Ricse,

E-mail: carlosricse@gmail.com importando (necesariamente) el costo. Es decir, es más importante conseguir impacto poblacional (eficacia) que lograr una maximización de los recursos escasos (eficiencia).

A principios de la década de 2000, el Perú todavía enfrentaba los estragos de la crisis recesiva internacional de finales de los noventa. No obstante, el Gobierno del Presidente Alejandro Toledo hizo esfuerzos importantes por aumentar el presupuesto de los sectores sociales y realizar reformas, impulsadas buena parte de ellas en compromisos con organismos multilaterales. Es así que el porcentaje de asignación presupuestal destinado a los sectores de salud, educación, nutrición, vivienda, saneamiento, tuvo una tasa de crecimiento anual de casi el doble de la tasa de crecimiento del Presupuesto Total del Sector Público. A eso se sumaron políticas de racionamiento de programas sociales, fortalecimiento de las encuestas oficiales de hogares, de protección presupuestaria, entre otras.

A pesar de este esfuerzo fiscal, no se obtuvo los resultados esperados. Es así, que entre el año 2000 y 2006, el gasto real en el sector educación se incrementó de S/. 3.97 mil millones a S/. 5.90 mil millones, es decir, un incremento real de $49 \%$. Los logros en cambio, no acompañaron este incremento de presupuesto: el nivel suficiente en comprensión de textos en los estudiantes de $6^{\underline{0}}$ de primaria, fue alcanzado en $8 \%$ de los que estudian en instituciones públicas y el 2,3\% de los estudiantes de escuelas pública rurales. En el caso de salud, la situación aunque de características diferentes, permite concluir de forma similar. En el mismo periodo el presupuesto para Salud Individual y Colectiva, pasó de S/. 1.66 mil millones a S/. 2,95 mil millones, es decir un incremento real de $78 \%$. A diferencia de lo encontrado en la educación pública, sí hubo mejoras en indicadores de mortalidad materna, mortalidad infantil, cobertura de parto institucional, entre otros. Sin embargo, los avances no fueron proporcionales al aumento de presupuesto y el Perú seguía por debajo de los promedios de América Latina, mostrando fuertes inequidades geográficas y económicas.

En vista de esta situación, a finales de 2005, las autoridades gubernamentales, en particular del Ministerio de Economía y Finanzas (MEF), tomaron en cuenta las recomendaciones y los compromisos con organismos multilaterales. Se establecieron en 
las Leyes de Presupuesto y Equilibrio Fiscal 2006 prioridades de política pública a las que se le daría preferencia en la asignación presupuestal durante el año 2006. Fueron 11 prioridades referidas a identidad, salud, nutrición, educación y saneamiento. En la práctica, durante el año 2006 no se logró revertir la tendencia del periodo 2000-2005.

En vista de que no se quebró la inercia anterior, en 2006, en el marco de la aprobación de las Leyes de Presupuesto, Endeudamiento y Equilibrio Fiscal para el año 2007, el Congreso incluyó el Capítulo IV de la Ley de Presupuesto, referido a la Implementación de Presupuesto por Resultados (PpR). En dicho capítulo se estableció el inicio de la implementación por las 11 prioridades declaradas el año anterior, y se incorporaron las evaluaciones y el monitoreo de indicadores no financieros, como parte de la metodología de implementación. Se encargó a la Dirección General de Presupuesto Público (DGPP) del MEF, la tarea de conducir el proceso de implementación progresiva.

La DGPP en un primer momento diseñó (hasta febrero 2007) una metodología que con base en los fundamentos de la Planificación Estratégica, establecería las bases para una asignación presupuestaria preferente y racional a las 11 prioridades. Sin embargo, luego de algunos cambios dentro de la DGPP, se varió la metodología a aquella que produjera los denominados Programas Estratégicos (PE). Los PE reunían resultados y productos ?con sus respectivos indicadores- como respuesta propositiva basada en evidencias, a los problemas y sus causas que estaban detrás de las 11 prioridades anteriores. La orientación de esta metodología PPE era la búsqueda de la eficacia, tratando de asegurar los resultados y productos en los beneficiarios, no importando los costos (en el corto plazo) ni la entidad que recibiera el presupuesto y los ejecutara.

La nueva metodología fue denominada como "Programación Presupuestaria Estratégica" (PPE) y a partir de su aplicación se diseñaron los primeros $5 \mathrm{PE}$ que explicitaban 5 prioridades de política pública (desnutrición infantil, muerte materna, logros educativos, identidad y transitabilidad terrestre) y más de medio centenar de productos, entre los que incluyeron las 11 prioridades mencionadas. Esta metodología PPE fue diseñada sobre la base de aquella utilizada en el Estudio de Preinversión de un proyecto de inversión pública del sector salud que se enfocaba en la muerte materna y en la desnutrición infantil. Cabe señalar que este estudio de preinversión se inició en 2005 y fue la fuente de información para la propuesta de 6 de las 11 prioridades de política citadas anteriormente.

Es importante señalar que esta metodología PPE difiere de aquella que el Banco Mundial y el Banco Interamericano de Desarrollo recomendaban usar, pues tomó las experiencias exitosas de otros países para su elaboración. Básicamente la recomendación era reforzar la gestión de indicadores no financieros, evaluaciones independientes e incentivos asociados. A diferencia de la PPE, la metodología recomendada priorizaba la eficiencia de la calidad del gasto, por encima de la eficacia.

En la DGPP, se dispuso que los 5 PE y los que se diseñaran, fueran incorporados progresivamente a la estructura presupuestal vigente, aunque forzando algunas definiciones. De este modo, en el presupuesto del año 2008, las entidades de los Gobiernos Nacional y Regional reordenarían su presupuesto para dar cabida a estos $5 \mathrm{PE}$, a los cuales se les destinó una asignación presupuestal adicional. En el marco de la implementación del PpR, además de la PPE fueron diseñadas las estrategias del monitoreo, evaluación, incentivos, capacitación y refuerzo normativo, para "incubar" su desarrollo, todo lo cual se empezó a ejecutar desde el $2^{0}$ semestre de 2007.

En el periodo comprendido entre 2008 y 2011, el sector público continuó avanzando en la implementación de $\mathrm{PpR}$, con el diseño de más $\mathrm{PE}$, la realización de evaluaciones independientes, el diseño de herramientas para la programación y seguimiento de la ejecución, el aumento de la asignación presupuestal (aunque sólo llegó a representar el 13.6\% del presupuesto total), la capacitación a operadores de presupuesto, entre los más importantes. Sin embargo, algunos pendientes fueron poco atendidos, lo que trajo consigo una evaluación de la implementación y la decisión de realizar ajustes a la metodología y a la velocidad de implementación del PpR. Entre los pendientes más resaltantes, y que motivaron cambios en 2011, está el que no se reconocían responsables ni productos institucionales, a los cuales ni por los cuales, pedir cuentas por el presupuesto asignado.

Es así que en 2011, durante el proceso de formulación presupuestal para el año 2012, la DGPP realiza un viraje en la metodología para el diseño de los programas de PpR. Suspende el uso de la metodología PPE y dispone su reemplazo por la metodología del Marco Lógico, reforzada por la exigencia de registrar evidencia empírica para sustentar la estructuración de resultados, productos y actividades. Dispone también que a partir de 2012 sean los Programas Presupuestales (PP) la expresión operativa de la implementación de PpR, en reemplazo de los anteriores Programas Estratégicos. Reforzando el cambio producido, el equipo de gobierno del Presidente Ollanta Humala, declara que al final de su periodo presidencial (julio de 2016) todo el presu- 
puesto público estará dispuesto bajo PpR.

Cabe recordar que el Perú es un país unitario y descentralizado, donde las políticas nacionales las definen los Rectores en el Gobierno Nacional, en tanto que los Gobiernos Regionales y Locales son implementadores y articuladores. El diseño de los $\mathrm{PP}$ se inicia, por lo tanto, con el reconocimiento de las funciones del Rector de las Políticas Nacionales, y de algún problema concreto que atender. De este modo, se estructuran los nuevos PP, para los cuales se necesitó rediseñar la estructura presupuestal (clasificación funcional programática). Por disposición de la DGPP, los rectores a los que pertenecían los PE existentes hasta 2011, rediseñarán los respectivos PP. Cabe resaltar que se prohibió el diseño de PP regionales o locales, reconociendo que su rol está en la programación y ejecución de productos y actividades ?de PP diseñados por el rector nacional- en su ámbito territorial.

Pero, ¿Por qué fue necesario realizar este cambio en la metodología de PpR?. Los PE priorizaban la eficacia ?por sobre la eficiencia-, sin embargo, se había dispuesto que sea la DGPP la conductora de su proceso de implementación, es decir a una dependencia del MEF cuya orientación por naturaleza institucional es la eficiencia. Este arreglo institucional hace crisis a finales de 2010 cuando el MEF comprueba que no había una respuesta proporcional al aumento presupuestal a PpR, en los indicadores de resultados y productos de los PE. Es decir una situación equivalente a lo ocurrido en el periodo 2000-2005. Entonces, la DGPP y el MEF en su conjunto, prefieren concentrarse en la rama de eficiencia de la calidad del gasto público, resultando en el diseño de los PP.

En el periodo comprendido entre 2012 y $1^{\underline{0}}$ semestre de 2013, se logró avanzar rápidamente en el tránsito hacia los PP, llegando a cerca del $50 \%$ del total del presupuesto. En 2014, el aumento ha sido marginal y se prevé situación similar para 2015. Pero el estancamiento del avance en el porcentaje del presupuesto no es el principal problema. Y es que subsisten los mismos problemas pendientes del diseño de los primeros $\mathrm{PE}$, los que no fueron abordados en 2011 con el cambio de metodología. Sea por la metodología PPE o la actual, existe un divorcio entre acciones permanentes e inversiones, la poca o nula incidencia en los procesos de la gestión pública distinta del presupuesto $\mathrm{y}$, sobre todo, el escaso abordaje de la articulación territorial de los esfuerzos públicos.

En relación al último pendiente señalado, al enfoque territorial de la gestión por resultados, cabe señalar que la responsabilidad en la función de producción de un servicio público en el Perú está dispersa entre múltiples entidades dependientes del Gobierno Nacional, Regional y Local. Como cada una tiene autonomía administrativa, presupuestal y financiera, y la Caja Fiscal está básicamente centralizada, todos terminan compitiendo por mayor asignación relativa, sin necesariamente estar preocupados por la integridad de la función de producción. La DGPP ha dispuesto que los PP consideren esfuerzos por la articulación territorial con dos grandes sesgos e inconvenientes: la articulación se hace dentro de cada PP por separado (no se permite la coordinación entre PP); no hay vínculo explícito entre las variables no financieras (indicadores de producto y resultado de los PP) y financieras, en particular en la etapa de ejecución presupuestal y financiera.

¿Qué está pasando con la implementación de PpR? ¿Ha fracasado? Cuando empezamos este desafío en 2007, los expertos internacionales que acudieron a asesorarnos nos alertaron que era un proceso largo y tortuoso. Que lo más difícil no eran los cambios en los sistemas administrativos y de información, sino en las personas y las instituciones a las que representan. A la vuelta de 7 años, no se equivocaron.

¿Qué ha ocurrido últimamente que pueda dar pistas que es un proceso en evolución positiva?. En primer lugar, se ha implementado este año 2014 (para la formulación presupuestal 2015) la programación multianual del presupuesto. En segundo lugar, se viene ejecutando experiencias piloto de vinculación entre la planificación up-down (distribución subnacional de compromisos nacionales) con la planificación down-up (customización de las tareas que deben hacerse para alcanzar los mismos productos de los PP), complementado con la vinculación de los planes resultantes (estratégicos y operativo) con el presupuesto expresado en los PP. En tercer lugar, se viene trabajando en la interoperabilidad entre los sistemas administrativos que respalden experiencias de gestión por resultados. En cuarto lugar, se vienen incorporando los proyectos dentro de la estructura presupuestal de los PP, entre los más importantes. Estos cambios, sin necesariamente representar el retorno al periodo 2008-2011, significan que se está equilibrando la eficacia con la eficiencia en la agenda de mejorar la calidad del gasto público en el Perú.

Por todo lo referido, es posible afirmar que se está gestando actualmente las bases para una nueva modificación en la metodología de implementación de PpR en el Perú. 